\title{
ON MINIMAL HYPERSURFACES OF NONNEGATIVELY RICCI CURVED MANIFOLDS
}

\author{
YOE ITOKAWA \\ Department of Mathematics \\ University of Alabama at Birmingham \\ Bi rmingham, Alabama 35294 \\ U. S. A.
}

(Received March 3, 1992 and in revised form June 1, 1992)

\begin{abstract}
We consider a complete open riemannian manifold $M$ of nonnegative Ricci curvature and a rectifiable hypersurface $\Sigma$ in $M$ which satisfies some local minimizing property. We prove a structure theorem for $M$ and a regularity theorem for $\Sigma$. More precisely, a covering space of $M$ is shown to split off a compact domain and $\Sigma$ is shown to be a smooth totally geodesic submanifold. This generalizes a theorem due to Kasue and Meyer.
\end{abstract}

KEY WORDS AND PHRASES. Riemannian manifold, nonnegative Ricci curvature, minimal hypersurface, integral currents.

1991 AMS SUBJECT CLASSIFICATION CODES. Primary 49Q15, 53C20; Secondary $53 \mathrm{C} 21$

\section{INTRODUCTION.}

Let $M$ be a complete connected riemannian manifold of dimension $d$ and class $C^{\infty}$. We assume that $M$ is noncompact and that the Ricci curvature of $M$ is everywhere nonnegative. In this case, A. Kasue [1] and D. Meyer [2] proved a statement from which the following structure theorem is inferred.

Suppose that there exists a smooth minimal immersion of a smooth compact connected $d-1$ dimensional manifold $T$ without boundary in $M$. Then, for some covering space $p: \tilde{M} \longrightarrow M$, there exists a compact region $Q \subseteq \tilde{M}$ possibly empty so that, depending on whether $Q$ is empty or not, $\underset{\sim}{\tilde{M}} \backslash Q$ splits isometrically as either the riemannian product $\tilde{T} \times \mathbf{R}$ or $\tilde{T} \times[0, \infty)$ where $\tilde{T}$ is the lifting of $T$ in $\tilde{M}$ by $p$.

However, the requirement that $T$ be smooth is not a natural one from the standpoint of minimal submanifold theory. In practice, for $d>7$, it is often unavoidable for minimal hypersurfaces to possess singularities. Our purpose in the present paper then is to remove this assumption from the theorem above. 
In this paper, by a compact hypersurface $\Sigma$, we mean an integral current in $M$ of Hausdorff dimension $d-1$. It is well known that the support, spt $\Sigma$, of such a $\Sigma$ is almost everywhere (with respect to Hausdorff measure) the union of a finite number of Lipschitz images in $M$ of compact $d-1$ dimensional orientable riemannian manifolds. In many ways, rectifiable currents are the correct setting for the theory of minimal submanifolds, because they are closed under taking weak limits. We refer to the books of Federer [3], Lawson [4], and Morgan [5]. We say that $\Sigma$ is without boundary if the $d-2$ dimensional current $\partial \Sigma$ is identically 0.

For an open set $U \subseteq M$, let $\Sigma \mid \bar{U}$ denote the localization of $\Sigma$ to the closure $\bar{U}$ of $U$. In this paper, we choose to call a current $\Sigma$ locally minimizing if, for each $x \in M$, there exists some neighborhood $U$ of $x$ in $M$ such that the varifold associated to $\Sigma \mid \bar{U}$ is absolutely mass minimizing for the variational problem supported on $U$. Although this condition is stronger than that of being merely stationary and is not a closed condition under the weak topology, it includes the solutions to most pertinent variational problems. Notice that it is weaker than the notion of locally minimizing as described by Lawson in [4] where $\Sigma$ is required to be absolutely minimizing with respect to all variations with compact support. In particular, our condition is satisfied by all weakly embedded smooth minimal submanifolds. Technically, our definition has the advantage of satisfying locally the the requirements for the regularity theorems of Almgren [6].

MAIN THEOREM. Suppose that there exists in $M$ a locally minimizing compact hypersurface $\Sigma$ without boundary in the sense described above. Then, for some covering space $p: \tilde{M} \longrightarrow M$, there exists a smooth compact orientable d-1 dimensional riemannian manifold $T$ and possibly empty compact region $Q \subseteq \tilde{M}$ such that $\tilde{M} \backslash Q$ splits isometrically as either the riemannian product $T \times \mathbf{R}$ or $T \times[0, \infty)$ and spt $p^{*} \Sigma$ consists of disjoint smoothly embedded copies of $T$.

Notice that in particular, our Main theorem implies the following regularity theorem.

COROLLARY. A locally minimizing compact hypersurface $\Sigma$ without boundary in a complete noncompact manifold $M$ of nonnegative Ricci curvature must be regular everywhere and totally geodesic.

In this sense, we point out its relation to the works of Anderson [7] and Anderson and Rodriguez [8].

of course, under our Main theorem, the Ricci curvature, in fact the sectional curvature, of $M$ in the direction of the 1 inear factor is 0 . Therefore, we obtain,

COROLLARY. Suppose that $M$ is a complete riemannian manifold of positive Ricci curvature. If there exists a minimal compact hyper- 
surface $\Sigma$ in $M$ without boundary, then $M$ itself must be compact.

This generalizes a part of our result in [9].

\section{Proof.}

Let $M$ be a complete connected noncompact riemannian manifold and let $d$ be its dimension. We recall from D. Gromoll and W. Meyer [10] that a ray in $M$ is a geodesic $c:[0, \infty) \longrightarrow M$ such that every segment $c \mid[0, b], 0<b$, is length minimizing. The function $b_{c}: M \longrightarrow R$ defined by

$$
b_{c}(p):=\lim _{s \rightarrow \infty} \rho(p, c(s))-s
$$

where $\rho$ is the distance function in $M$ is called the Busemann function associated to $c$.

In [11], J. Cheeger and Gromoll prove that $b_{c}$ is always continuous and that if $M$ has nonnegative Ricci curvature, then $b_{c}$ is superharmonic. Moreover, Galloway [12] proves that $T$ is any piece of smooth minimal hypersurface of $M$, then $b_{c} \mid T$ obeys the Strong minimum principle; i.e. .

If $U \subset T$ is a relatively open connected set of $T$ and $b_{c}$ attains a local minimum value in $U$, then $b_{c}$ is constant throughout $U$.

Actually, the result, Lemma (2.4) in [12], is stated for the case $M$ is a Lorentzian spacetime with positive timelike Ricci curvature, but the same proof goes through for our case. Cf. also a related result of Galloway and Rodriguez [13].

LEMMA 1. Let $A \subseteq M$ be any compact set. Then, for each end $E$ of $M$ there exists a ray $c$ in $M$ such that $c(0) \in A, c(s) \in E$ for large $s$, and $b_{c}$ is nonnegative on $A$.

Here, by $c(s) \in E$ for large $s$, we mean that for each component $E_{i}$ of the complement of the filtration defining the end $E$, there is a $t>0$ such that $c(s) \in E_{i}$ for all $s>t$.

PROOF. Take a sequence of points $q_{i}$ in $E$ such that $\rho\left(A, q_{1}\right)$ $>1 / i$. Since $A$ is assumed to be compact, for each $i$, there is a unit-speed geodesic segment $c_{i}:\left[0,1_{i}\right] \longrightarrow M$ realizing the distance between $A$ and $q_{1}$. Let $\tau_{1}:{ }_{1} T M \longrightarrow M$ be the unit tangent sphere bundle of $M$. Then, $c_{i}(0) \in \tau_{1}^{-1}(A)$ while the latter is a compact space and so the sequence of tangent vectors $\dot{c}_{i}(0)$ has some subsequence converging to a vector $u \in \tau_{1}^{-1}(A)$. Then, $c(s):=\exp s u$ is a ray with $c(0) \in A$ and $c(s) \in E$ for large $s$. Moreover, if we define a sequence of functions $\delta_{i}: M \longrightarrow R$.

$$
\boldsymbol{g}_{i}(p):=\rho\left(p, q_{i}\right)-1_{i},
$$

then, $\boldsymbol{g}_{i}$ converge pointwise to the Busemann function $b_{c}$. Since $\boldsymbol{g}_{i} \mid A$ is nonnegative, we obtain the lemma.

LEMMA 2. Lèt $c$ be a ray constructed for a compact set $A$ as in Lemma 1. Then, $q:=c(0)$ is the closest point in $A$ to any point on 
the ray $c$.

PROOF. Let $x:=c(t)$. Assume that there is a point $y \in A$ which is closer to $x$ than $q$. Then, $\rho(y, x)=t-\varepsilon$ for some $\varepsilon>0$. Hence, for all $s>t$.

$$
\rho(y, c(s)) \leq \rho(y, x)+\rho(x, c(s))=t-\varepsilon+s-t=s-\varepsilon .
$$

It follows that

$$
b_{c}(y)=\underset{s \rightarrow \infty}{1 \operatorname{im} \rho} \rho(y, c(s))-s \leq-\varepsilon,
$$

which contradicts Lemma 1.

We mention that Shioya [14] made the same observations as our two preceding lemmas but used them for a different purpose.

Now, let $\Sigma$ be a locally minimizing compact hypersurface in $M$ and let $A$ be the support of $\Sigma$. Construct a ray $c$ associated to $A$ as in Lemma 1 . We recall an observation of $M$. Gromov [15] that for any point $x \notin A$, the point $y \in A$ which is closest to $x$ is a regular point of $\Sigma$ in the sense that there is a neighborhood $U$ of $q$ in $M$ so that spt $(\Sigma \mid U)$ is a smoothly embedded image of a piece of $d-1$ dimensional smooth manifold. Therefore, by virtue of Lemma 2, we see,

LEMMA 3. $q:=c(0)$ is a regular point of $\Sigma$.

Let $S$ be the connected component of $q$ in spt $\Sigma$ and $W$ the smoothly embedded piece of $d-1$ manifold defining $\Sigma \mid U$ in a neighborhood $U$ of $q$. We may assume that $W \leq S$. We can then apply the lemma of Galloway and Rodriguez to conclude that $b_{c} \mid w$ obeys the Strong minimal principle. But, $b_{c} \mid S \geq 0$ and $b_{c}(q)=0$, so $q$ is a minimum point of $b_{c}$ in $S$. Moreover, $q$ is an interior point of $W$, so we conclude that $b_{c}=0$ on $W$. But since $b_{c}$ is continuous and $S$ is connected, $b_{c}$ is in fact identically 0 on $S$. In particular, $S$ is regular everywhere. Moreover, by the techniques of Kasue [1] and Meyer [2], this induces a local splitting of $M$; i.e., M contains a region $R$ in turn containing $W \cap R$ as part of its boundary and $R$ splits isometrically as the product $(W \cap R) \times[0, \infty)$.

Now, we recall that for purely topological reasons, there is a covering space $p: \tilde{M} \longrightarrow M$ of $M$ so that $p^{-1}(S)$ decomposes into a finite number of connected components each of which, say $T$, divides $\tilde{M}$ itself into two connected regions with $T$ as its common boundary. Then, it is easily seen that, passing to $\tilde{M}$, the splitting of $p^{-1}(R)$ extends to the global splitting $T \times[0, \infty)$.

Now, let $P$ be the region in $\tilde{M}$ isometric to $T \times[0, \infty)$ and let $Q:=\tilde{M} \backslash P ;$ If the closure of $Q$ is not compact, then $Q$ contains an end $E^{\prime}$ of $\tilde{M}$. We can construct a ray as in Lemma 1 for the compact set $T$ and argue as before to conclude that $Q$ also splits as $T \times(-\infty, 0]$. Otherwise, the closure of $Q$ has to compact. This proves the first half of our Main theorem. 
Now, suppose that spt $p^{*} \Sigma$ contains a connected component $T \neq T$. This time, we can find a geodesic segment $\gamma:[0, k] \longrightarrow \tilde{M}$ realizing the distance between the two compact sets $T$ and $T$. Say $\boldsymbol{\gamma}(0) \in T$ and $\boldsymbol{\gamma}(\boldsymbol{k}) \in T$. Then, by Gromov's lemma again $\boldsymbol{\gamma}(0)$ is also a regular point of $p^{*} \Sigma$. Moreover, since $T$ is now a smooth totally geodesic submanifold, the function $g: M \longrightarrow R_{+} \cup\{0\}$ defined by

$$
g(p):=\rho(p, T)
$$

is superharmonic on $M$. Again, by Lemma (2.4) of Galloway [12], there is a neighborhood $V$ of $\gamma(0)$ in $T$ so that $g \mid V$ obeys the Strong minimal principle. By repeating the previous argument again, we obtain that there is a region in $\tilde{M}$ bounded by $T \cup T$ and isometric to $T \times[0, k]$. In particular, $T^{\nu}$ is isometric and parallel with respect to the original Busemann function to $T$. Since spt $p^{*} \Sigma$ is compact and can contain only finitely many connected components, by induction, this completes the proof of the Main theorem.

\section{REMARKS.}

Geometric measure theory seem to provide us with useful techniques in studying global properties of riemannian manifolds. We mention a possible application of our result to the following classical,

CONJECTURE. Suppose that $M$ is a complete noncompact manifold with positive Ricci curvature. Then, the $(d-1)$ st. integral homology class $H^{d-1}(M, Z)=\{0\}$.

Recently, Shen [16] proved the conjecture with the additional assumption that $M$ is proper. However, this is a strong assumption.

With a little more work, we can show that our splitting theorem holds for $\Sigma$ which is not a priori minimal but is homologically nontrivial and a solution to a certain elliptic variational problem with constraint on the $d$-dimensional measure of $\Gamma$ where $\partial \Gamma=\Sigma+\Sigma^{\prime}$ for some other compact hypersurface $\Sigma^{\prime}$. For the case $M$ is compact, it is well known that each element of $H_{*}(M, Z)$ is in fact represented by a locally area minimizing compact hypersurface. Unfortunately, the .weak convergence of integral currents has not been applied much to noncompact manifolds. However, heuristically, it seems to us that, in many cases, the existence of such $\Sigma, \Sigma^{\prime}$, and $\Gamma^{\prime}$ is a common occurrence. Whenever such existence can be established, therefore, our result implies a partial solution to the conjecture.

ACKNOFLEDGEMENT. We would $1 \mathrm{ike}$ to thank M. T. Anderson and N. Nakauchi for helpful conversations during this work. We are also grateful to G. Galloway for pointing out an error in the original version of this paper and telling us how it may be fixed. 


\section{REFERENCES}

1. KASUE, A. Ricci curvature, geodesics and some geometric properties of riemannian manifolds with boundary, J. Math. Soc. Japan 35 (1983), 117-131.

2. MEYER, D. Sur les hypersurfaces minimales des variétés à curbure de Ricci positive ou nulle, Bull. Soc. Math. France 111 (1983), 359-366.

3. FEDERER, H. Geometric Measure Theory. New York: Springer Verlag, 1969.

4. LAWSON. H. B. Minimal Yarieties in Real and Complex Geometry. Montreal: L'Universite de Montreal, 1974.

5. MORGAN, F. Geometric Measure Theory, A Beginner's Guide. Boston: Academic Press, 1988.

6. ALMGREN, F. J. Jr. Existence and regularity almost everywhere of solutions to elliptic variational problems, Mem. Amer. Math. Soc. 4:165 (1976).

7. ANDERSON, M. T. On area-minimizing hypersurfaces in manifolds of non-negative curvature, Indiana Univ. Math. J. 32 (1983), 745760.

8. ANDERSON, M.T. and RODRIGUEZ, L. Minimal surfaces and 3-manifolds of non-negative Ricci curvature, Math. Ann. 284 (1989), 461-475.

9. ITOKAWA, Y. Distance spheres and Myers-type theorems for manifolds with lowerr bound on the Ricci curvature, Illinois $\mathrm{J}$. Math. 34 (1990), 693-705.

10. GROMOLL, D. and MEYER, W. On complete open manifolds of positive curvature, Ann. of Math. 90 (1969), 75-90.

11. CHEEGER, J. and GROMOLL, D. The splitting theorem for manifolds of nonnegative Ricci curvature, J. Differential Geom. 6 (1971), 119-128.

12. GALLOWAY, G. J. The Lorentzian splitting theorem without completeness assumption, J. Differential Geom. 29 (1989). 373389.

13. GALLOWAY, G. J. and RODRIGUEZ, L. Intersections of minimal submanifolds, Geom. Dedicata 39 (1991), 29-42.

14. SHIOYA, $T$. The behavior of distant maximal geodesics in a complete riemannian plane with total curvature, Preprint, Kyushu University.

15. GROMOV, M. Paul Levy's isoperimetric inequality, Preprint, I.H. E. S. , Paris.

16. SHEN, $Z$. Finite topological type and vanishing theorems for riemannian manifolds, Thesis, State University of New York at Stony Brook, 1990. 


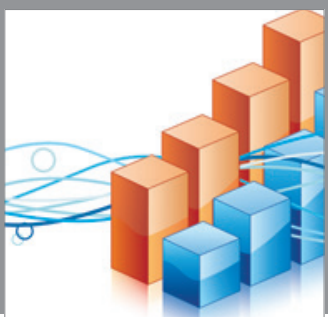

Advances in

Operations Research

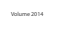

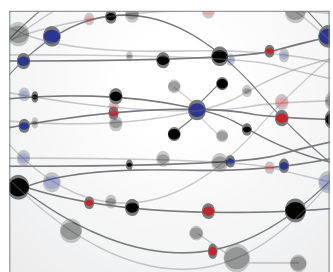

\section{The Scientific} World Journal
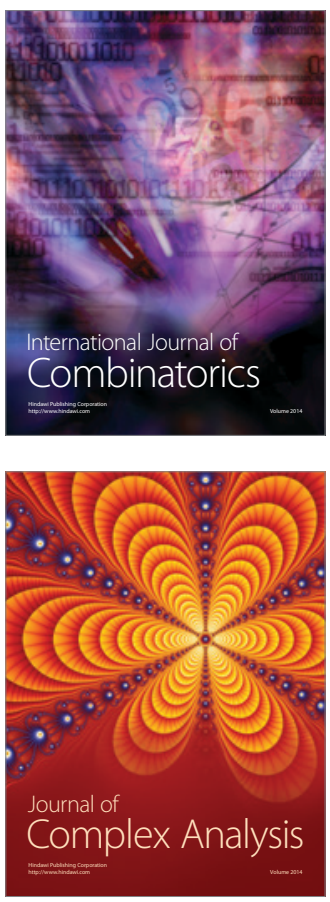

International Journal of

Mathematics and

Mathematical

Sciences
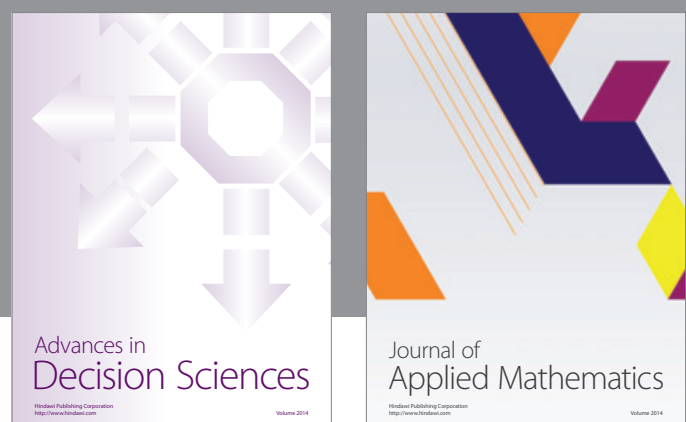

Journal of

Applied Mathematics
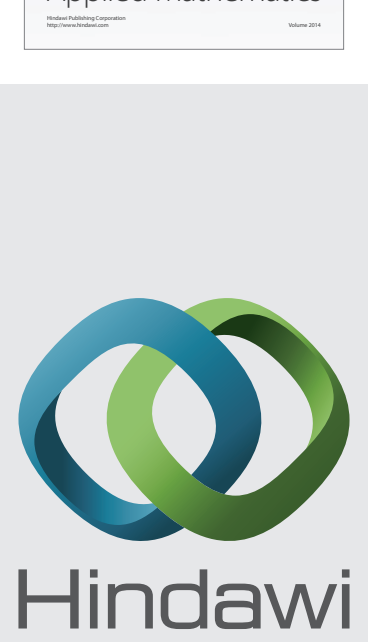

Submit your manuscripts at http://www.hindawi.com
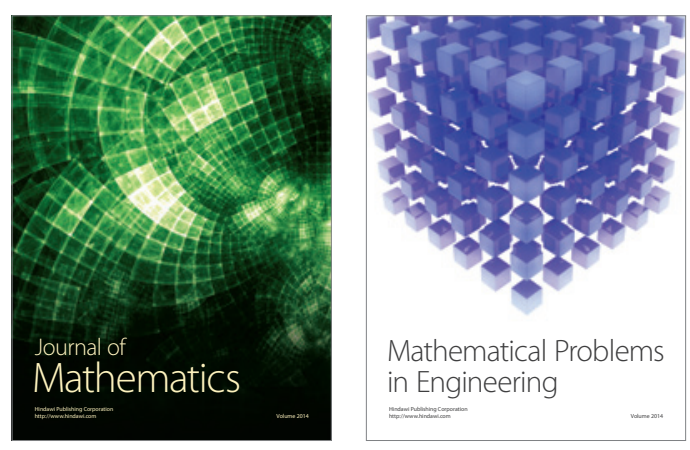

Mathematical Problems in Engineering
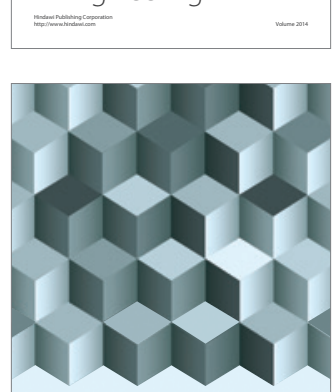

Journal of

Function Spaces
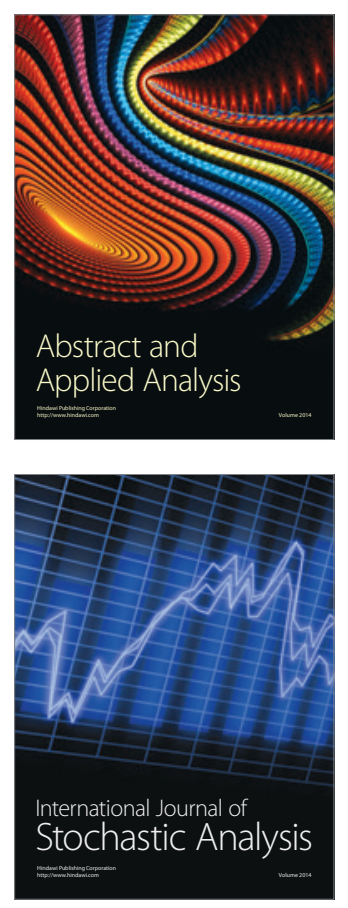

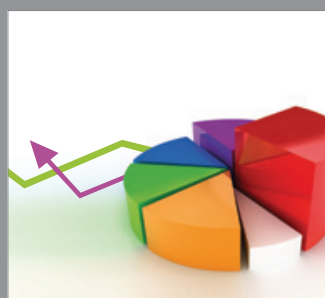

ournal of

Probability and Statistics

Promensencen
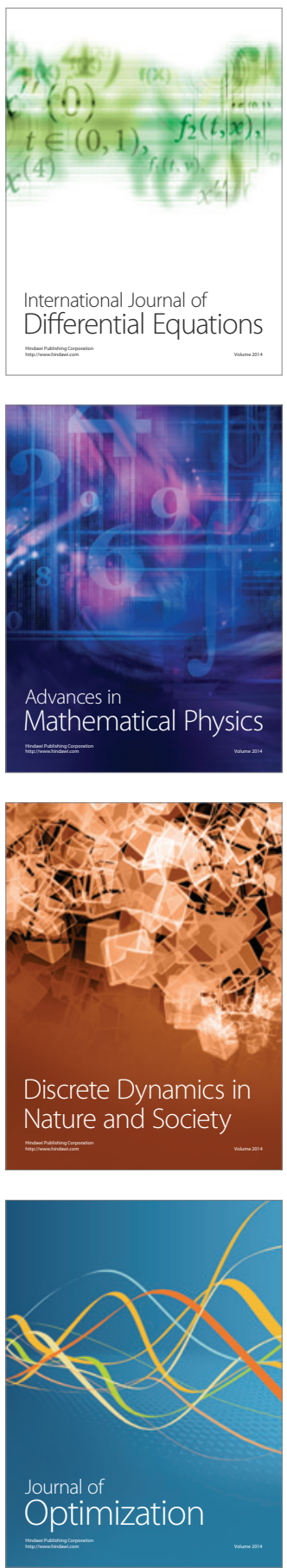